\title{
Nanoparticles Mediated Drug Delivery
}

Nida Tabassum Khan *

Department of Biotechnology, Balochistan University of Information Technology Engineering and Management Sciences (BUITEMS), Quetta, Pakistan

*Corresponding author: Nida Tabassum Khan, Department of Biotechnology, Balochistan University of Information Technology Engineering and Management Sciences (BUITEMS), Quetta, Pakistan, Tel: +03368164903; E-mail: nidatabassumkhan@yahoo.com

Received date: July 27, 2017; Accepted date: August 08, 2017; Published date: August 14, 2017

Copyright: (C) 2017 Khan NT. This is an open-access article distributed under the terms of the Creative Commons Attribution License, which permits unrestricted use, distribution, and reproduction in any medium, provided the original author and source are credited.

\begin{abstract}
The term "nano" has originated from Greek word meaning extremely small or dwarf. Nanoparticles are nano-sized objects whose size is measured in nanometers $(\mathrm{nm})$ ranging from $0.1-100 \mathrm{~nm}$ exhibiting distinct morphological characteristics which is quite different from their bulk form.
\end{abstract}

Keywords: Nano scale; Nano carriers; Quantum dot

\section{Introduction}

"Nanotechnology" the term was coined by Taniguchi [1]. It implies manipulation, reduction and fabrication of materials at nano scale with distinctive properties such as good strength, cost effective, lighter, ecofriendly, definite and specific etc and enhanced functionality and improved stability [2]. Nanotechnology has a wide range of applications; one of its potentials has been recognized in drug delivery systems for prevention and therapy of different diseases. Nanoparticles have been modified to effectively deliver drugs to the target regions in the human body. Besides, drug accumulation and its metabolism can also be tracked in the cells or tissues by using these tiny entities to get a better understanding of pharmacokinetics and pharmacodynamics of a particular drug [3].

\section{Properties of Nanoparticles that Made Them Suitable for Drug Delivery}

Nanoparticles being target specific have been used to deliver chemotherapeutics targeted to the tumor tissue without damaging normal organs $[4,5]$.

Nanoparticles are chemically stable structures that could be fabricated through different chemical and biological routes that are environment friendly and cost effective.

These entities are designed in such a way that can easily diffuse into the cell by interacting with specific cellular components to permit selective targeting and accumulation in specific cell or tissues. Besides nanoparticles are $\mathrm{pH}$-labile structures that easily degrades at low $\mathrm{pH}$ in the cell to release drug payloads. Examples of few biodegradable nanoparticles used for drug delivery system includes polymeric polyethylene-glycol etc. Nanoparticles can persist in the micro fluid environment of the cell for weeks or more without being degraded. Thus make them suitable for drug delivery systems. For example carbon nanotube can persist for a longer period of time with extensive functionalization and loading of cargo. Nanoparticles are designed in such a way that they are non-immunogenic i.e., compatible with the body immune system. As a result will not engage in initiating an immune response when introduced in the body [6].

\section{Types of Nanoparticles for Drug Delivery}

Numerous different types of nanostructures exhibiting different physiochemical properties are employed to improve the efficiency of drug delivery to specific targets $[7,8]$. Few examples are given below:

\section{Polymerosomes}

Polymersome nanoparticles are synthetic amphiphilic blocks that are spherical vesicular bodies containing an aqueous solution. This unique copolymer is made of two components, a hydrophobic and hydrophilic subunits e.g. joined together. Poly lactic/glycolic acid, Polyethylene glycol-block-polycaprolactone etc. $[9,10]$.

\section{Nanocapsule}

Are also known as liposomes which are a lecithin and stearate encapsulated structure with a lipid core where lecithin is sited in the inner part of the capsule. Polyethylene glycol could be employed to increase its half-life $[11,12]$.

\section{Silica nanoparticles}

These are mesoporous nanoparticles with high specificity in terms of pore volume and surface area. Differential surface enhancements are employed to optimize particle size which directly influences its function. Thus making them suitable to be used in imaging tools [9].

\section{Quantum dot}

Amalgamated nanoparticles composed of two subunits Poly L-lactic acid-block-polyethylene glycol. High optical resolution, small size and property of fluorochrome made them useful for tracing the dynamics of many biological molecular pathways [13]

\section{Amphiphilic nanoparticles}

These dual nature nanoparticles consisted of hydrophobic and hydrophilic regions. Hydrophobic regions provide protection against the polar surroundings forming a micelle. In case of drug delivery it carries the drug and provides protection from the body's immune system to prevent its elimination e.g. polyethylene oxide-polyaspartic acid copolymers micelle carrying the drug doxorubicin [14]. 


\section{Dendrimers}

Repeatedly branching nanostructures with highly specific large surface area making them target specific. It is mostly composed of polyamidoamines [15,16]. For example telodendrimers made of polyethylene glycol and dendritic cholic acid subunits [17,18].

\section{Graphene}

Allotropic form of carbon with unique thermochemical properties and high tensile strength. Its unique hexagonal structure enables infrared radiation conversion in to heat. High surface area allows drug delivery in cancerous cell but is quite toxic in nature therefore requires surface shielding $[19,20]$.

\section{Carbon nanotubes}

These are basically graphene nanotubes used as hybrid drug carries. When bombarded with near-infrared radiation, it allows controlled drug release at specific tumor sites to protect the neighboring normal tissues [21-23].

\section{Metal-core nanoparticles}

Such nano entities are magnetic and photothermally stable. In response to an external magnetic field these entities vacillates and heat up e.g. Magnetite and maghemite [24].

\section{Oligopeptides}

$\mathrm{pH}$ sensitivity zwitterionic oligopeptide lipids nanoparticles mostly used as nanocarriers [25].

\section{Supramolecularpolymers}

Due to their reversible monomer-to-polymer transitions efficient in drug delivery [26].

\section{Drug Delivery Targeting}

There are three main methods to transport drug-loaded nanoparticles to diseased sites:

\section{Passive targeting}

Enhanced permeation and retention effect is the mechanism behind passive targeting which is the property of tumor cells to absorb particles of specific size than normal cells due to drippy tumor blood vessels and faulty particle screening [27]. Nanoparticle morphology and electrochemical properties influence enhanced permeation and retention effect thus effecting drug penetration, duration of circulation and intracellular stability $[28,29]$.

\section{Active targeting}

Active targeting involves the use of ligands such as antibodies, proteins, and peptides bound to nanoparticle surface to increase their uptake selectivity by interacting with the overexpressed receptors at the target sites [30]. These ligands often protect nanoparticles from enzyme destruction. High binding affinity of ligands to the target cell will increase drug delivery efficiency [31]

\section{Physical targeting}

Physical targeting uses external sources or fields to guide the nanoparticle to the target site and also controls the release process, for example, in photothermal and magnetic hyperthermia therapy [32].

\section{Challenges in nanoparticle designing for drug delivery}

Many challenges have been encountered when designing medically significant nanoparticles for drug delivery. The classified and nonuniform nature of nanoparticles is a prime challenge encountered during nanoparticle designing. A small alteration in a single existing property can have deleterious consequences on the pharmacokinetics and pharmacodynamics of the particle [33]. For example maintaining a narrow distribution of particle size is an issue. Particle size under 200 $\mathrm{nm}$ is desirable but with a broad normal distribution it is difficult to limit the number of particles over $200 \mathrm{~nm}$ [34]. In addition, variation in nanoparticle structure must be smaller otherwise the designed nanoparticle works in a lab setting will not work in an actual cellular environment. Nanoparticle stability is one of the major concerns that it should be stable in solution and should not aggregate over time. Finally, nanoparticles toxicity should be minimized because it greatly increases the time and cost of clinical trials. These challenges must be overcome in order to frame nano entities with enhanced properties thus making them suitable for drug delivery [35].

\section{Conclusion}

Nanoparticles are excellent candidates for targeted drug delivery. Being stably biocompatible and readily available allows for precise doses of drugs to be encapsulated and delivered directly to the target site. However nanoparticles morphology, electrochemical and thermal properties should be controlled to avoid side effects. Environmental factors can also impact nanoparticle drug delivery efficacy. Therefore a nanoparticle aimed for drug delivery must undergo clinical trials before practical application to ensure that they do not cause harm to living tissue or animals.

\section{References}

1. Taniguchi N (1974) On the basic concept of nano-technology. In Proc Intl Conf Prod London 1974. British Society of Precision Engineering.

2. De Jong WH, Borm PJ (2008) Drug delivery and nanoparticles: applications and hazards. Int J Nanomed 3: 133.

3. Kimura S, Egashira K, Chen L, Nakano K, Iwata E, et al. (2009) Nanoparticle-mediated delivery of nuclear factor $\mathrm{\kappa B}$ decoy into lungs ameliorates monocrotaline-induced pulmonary arterial hypertension. HTN 53: 877-883.

4. Ochekpe NA, Olorunfemi PO, Ngwuluka NC (2009) Nanotechnology and drug delivery part 1: background and applications. Trop J Pharm Res 8: 3 .

5. Poste G, Kirsh R (1983) Site-specific (targeted) drug delivery in cancer therapy. Nature Biotechnol 1: 869-878.

6. Dobrovolskaia MA, McNeil SE (2007) Immunological properties of engineered nanomaterials. Nat Nanotechnol 2: 469-478.

7. Ranjan S, Sood R, Dudas J, Glueckert R, Schrott-Fischer A, et al. (2012) Peptide-mediated targeting of liposomes to TrkB receptor-expressing cells. Int J Nanomed 7: 3475.

8. Steketee MB, Moysidis SN, Jin XL, Weinstein JE, Pita-Thomas W, et al. (2011) Nanoparticle-mediated signaling endosome localization regulates growth cone motility and neurite growth. Proc Natl Acad Sci USA 108: 19042-19047. 
9. Pritz CO, Dudás J, Rask-Andersen H, Schrott-Fischer A, Glueckert R (2013) Nanomedicine strategies for drug delivery to the ear. Nanomed 8 1155-1172.

10. Roy S, Johnston AH, Newman TA, Glueckert R, Dudas J, et al. (2010) Cell-specific targeting in the mouse inner ear using nanoparticles conjugated with a neurotrophin-derived peptide ligand: potential tool for drug delivery. Int J Pharm 390: 214-224.

11. Bastiat G, Pritz CO, Roider C, Fouchet F, Lignières E, et al. (2013) A new tool to ensure the fluorescent dye labeling stability of nanocarriers: a rea challenge for fluorescence imaging. J Control Release 170: 334-342.

12. Uster PS, Allen TM, Daniel BE, Mendez CJ, Newman MS, et al. (1996) Insertion of poly (ethylene glycol) derivatized phospholipid into preformed liposomes results in prolonged in vivo circulation time. FEBS Lett 386: 243-246.

13. Pathak S, Cao E, Davidson MC, Jin S, Silva GA (2006) Quantum dot applications to neuroscience: new tools for probing neurons and glia. J Neurosci 26: 1893-1895.

14. Kwon GS, Yokoyama M, Okano T, Sakurai Y, Kataoka K (1993) Biodistribution of micelle-forming polymer-drug conjugates. Pharm Res 10: 970-974

15. Svenson S, Tomalia DA (2012) Dendrimers in biomedical applicationsreflections on the field. Adv Drug Deliv Rev 64: 102-115.

16. Patri AK, Myc A, Beals J, Thomas TP, Bander NH, et al. (2004) Synthesis and in vitro testing of $\mathrm{J} 591$ antibody-dendrimer conjugates for targeted prostate cancer therapy. Bioconjug Chem 15: 1174-1181.

17. Wang Y, Gao S, Wen-Hui Y, Yoon HS, Yi-Yan Y (2006) Co-delivery of drugs and DNA from cationic core-shell nanoparticles self-assembled from a biodegradable copolymer. Nat Mater 5: 791.

18. Li Y, Xiao K, Luo J, Xiao W, Lee JS, et al. (2011) Well-defined, reversible disulfide cross-linked micelles for on-demand paclitaxel delivery. Biomaterials 32: 6633-6645.

19. Wang Y, Wang K, Zhao J, Liu X, Bu J, et al. (2013) Multifunctional mesoporous silica-coated graphene nanosheet used for chemophotothermal synergistic targeted therapy of glioma. J Am Chem Soc 135: 4799-4804.

20. Sahu A, Choi WI, Lee JH, Tae G (2013) Graphene oxide mediated delivery of methylene blue for combined photodynamic and photothermal therapy. Biomaterials 34: 6239-6248.

21. Estrada AC, Daniel-da-Silva AL, Trindade T (2013) Photothermally enhanced drug release by $\kappa$-carrageenan hydrogels reinforced with multiwalled carbon nanotubes. RSC Adv 3: 10828-10836.
22. Zhang H, Chen C, Hou L Jin N, Shi J, et al. (2013) Targeting and hyperthermia of doxorubicin by the delivery of single-walled carbon nanotubes to EC-109 cells. J Drug Targeting 21: 312-319.

23. Prato M, Kostarelos K, Bianco A (2007) Functionalized carbon nanotubes in drug design and discovery. Acc Chem Res 41: 60-68.

24. Thiesen B, Jordan A (2008) Clinical applications of magnetic nanoparticles for hyperthermia. Int J Hyperthermia 24: 467-474.

25. Mo R, Sun Q, Li N, Zhang C (2013) Intracellular delivery and antitumor effects of $\mathrm{pH}$-sensitive liposomes based on zwitterionic oligopeptide lipids. Biomaterials 34: 2773-2786.

26. Aida T, Meijer EW, Stupp SI (2012) Functional supramolecular polymers. Science 335: 813-817.

27. Matsumura Y, Maeda H (1986) A new concept for macromolecular therapeutics in cancer chemotherapy: mechanism of tumoritropic accumulation of proteins and the antitumor agent smancs. Cancer Res 46: 6387-6392.

28. Maeda H, Sawa T, Konno T (2001) Mechanism of tumor-targeted delivery of macromolecular drugs, including the EPR effect in solid tumor and clinical overview of the prototype polymeric drug SMANCS. J Control Release 74: 47-61.

29. Yuan F, Dellian M, Fukumura D, Leunig M, Berk DA, et al. (1995) Vascular permeability in a human tumor xenograft: molecular size dependence and cutoff size. Cancer Res 55: 3752-3756.

30. Bertrand N, Wu J, Xu X, Kamaly N, Farokhzad OC (2014) Cancer nanotechnology: the impact of passive and active targeting in the era of modern cancer biology. Adv Drug Deliv Rev 66: 2-25.

31. Ruoslahti E (2002) Specialization of tumour vasculature. Nat Rev. Cancer 2: 83 .

32. Huang X, Jain PK, El-Sayed IH, El-Sayed MA (2008) Plasmonic photothermal therapy (PPTT) using gold nanoparticles. Lasers Med Sci 23: 217.

33. Igarashi E (2008) Factors affecting toxicity and efficacy of polymeric nanomedicines. Toxicol Appl Pharmacol 229: 121-134.

34. Li G, Song S, Guo L, Ma S (2008) Selfassembly of thermoand pHresponsive poly(acrylic acid)bpoly(Nisopropylacrylamide) micelles for drug delivery. J Polym Sci A Polym Chem 46: 5028-5035.

35. Desai (2012) Challenges in development of nanoparticle-based therapeutics. AAPS J 14: 282-295. 